\title{
AN EXPERIMENTAL SIMULATION OF THE CULTURAL TRANSMISSION OF PRESTIGE AND DOMINANCE SOCIAL RANK CUES
}

\author{
Ángel V. Jiménez \& Alex Mesoudi \\ Human Behaviour and Cultural Evolution Group (HuBCEG) \\ Department of Biosciences \\ University of Exeter Cornwall Campus \\ Penryn, Cornwall TR10 9FE, United Kingdom
}

Correspondence should be addressed to Ángel V. Jiménez: aj419@exeter.ac.uk

15

6

\section{(7)}




\section{ABSTRACT}

Informal social hierarchies within small human groups are argued to be based on prestige, dominance, or a combination of these two (Henrich \& Gil-White, 2001). Prestige-based hierarchies entail the ordering of individuals by the level of admiration and respect they receive from others due to their competence within valued domains. This type of hierarchy provides benefits for subordinates such as high-quality social learning opportunities and both private and public goods. In contrast, dominance-based hierarchies entail the ordering of individuals by their capacity to win fights, coerce and intimidate others. This type of hierarchy produces costs in subordinates due to its aggressive and intimidating nature. Given the benefits and costs associated with these types of social hierarchies for subordinates, we hypothesized that prestige and dominance cues are better recalled and transmitted than social rank cues that do not elicit high prestige or dominance associations (here medium social rank cues). Assuming that for the majority of the population who are not already at the top of the social hierarchy it is more important to avoid the costs of dominance-based hierarchies than to obtain the benefits of prestige-based hierarchies, we hypothesized that dominance cues are better transmitted than prestige cues. We conducted a recall-based transmission chain experiment with 30 chains of four generations each $(\mathrm{N}=120)$. Participants read and recalled three descriptions of prestigious, dominant and medium social rank footballers, and their recall was then passed to the next participant within their chain. As predicted, we found that both prestige cues and dominance cues were better transmitted than medium social rank cues. However, we did not find support for our prediction of the better transmission of dominance cues over prestige cues. We discuss whether the results might be explain by a specific social-rank content transmission bias or by a more general emotional content transmission bias.

KEYWORDS: PRESTIGE, DOMINANCE, STATUS, SOCIAL RANK, CULTURAL EVOLUTION, TRANSMISSION BIASES, SOCIAL LEARNING, TRANSMISSION CHAIN 


\section{1.- INTRODUCTION}

Social hierarchies are a universal phenomenon in our species (Von Rueden, 2014), emerging rapidly and spontaneously during social interactions (Anderson \& Kilduff, 2009; Cheng, Tracy, Foulsham, Kingstone, \& Henrich, 2013; Smith \& Foti, 1998). Being at the top of the hierarchy in a human social group is associated with positive fitness outcomes such as greater access to resources, mating opportunities and greater number of surviving offspring (Betzig, 1988; Chagnon, 1988; Hill, 1984; Mealey, 1985; Savin-Williams, 1979; Snyder, Kirkpatrick, \& Barrett, 2008; Von Rueden, 2014; von Rueden, Gurven, \& Kaplan, 2010; von Rueden \& Jaeggi, 2016). People at the top usually act as leaders of groups, which helps to solve group problems such as collective decision-making and within-group coordination (Anderson \& Willer, 2014). Consequently, social hierarchy is not only beneficial for the individuals at the top but also, potentially, for groups and their members. Nevertheless, hierarchies also produce costs for groups and their members as they can lead to abuse of lower social rank individuals, which diminishes group morale and lowers group performance (Anderson \& Willer, 2014).

The dual evolutionary model of social hierarchy (Cheng, 2019; Cheng \& Tracy, 2014; Cheng et al., 2013; Henrich \& Gil-White, 2001; Jiménez \& Mesoudi, 2019, December 27; Redhead, Cheng, \& O'Gorman, 2018a) might help to explain this contrast between the benefits and costs of social hierarchy. According to this model, informal social hierarchies within small human groups can be based on prestige, dominance, or a combination of the two. Prestige-based hierarchies entail the ordering of members of a social group by their prestige (sometimes referred to as 'status'), which is defined as the respect, esteem and admiration that they receive by other members of the group (Anderson, Hildreth, \& Howland, 2015). Prestige is given by others (Blader \& Chen, 
2014; de Waal-Andrews, Gregg, \& Lammers, 2015), usually due to being competent in domains that are appreciated by a group (Henrich \& Gil-White, 2001). For example, frequently scoring goals is an indicator of competence in football, which is highly regarded by people who play/like football. As such, top goal scorers tend to attain prestige within football teams. However, competence in itself might often not be enough to be granted prestige. Competence needs to be acknowledged by others (e.g., by having many social connections) and these others have to expect to benefit from that competence somehow. In our example of football players, it would be difficult for an excellent football player to be conferred prestige if he/she is never seen playing or if he/she plays well but he/she plays in a rival team or does not share tips about his/her football skills. Therefore, prestige-biased hierarchies are not only meritocratic, but also beneficial for less knowledgeable/skilful individuals as being close to prestigious individuals gives them opportunities to social learners to acquire valuable knowledge/skills (Henrich \& Gil-White, 2001) and being provided with private (Pinker, 1998, p. 499) and public (Price \& Van Vugt, 2014) goods.

In contrast, dominance-based hierarchies entail the ordering of individuals in the capacity to win fights, coerce and intimidate others (Redhead, Cheng, \& O'Gorman, 2018b). Dominance is a form of informal social rank that is imposed upon others (de Waal-Andrews et al., 2015; Henrich \& Gil-White, 2001), sometimes with the help of coalitional alliances. Consequently, the top of dominance-based hierarchies are usually occupied by individuals who possess physical and material characteristics that are useful to win fights and intimidate others, such as having greater strength, higher fighting skills, greater coalitional support, and the possession of more and/or better weapons or other resources that could be used to inflict costs. Therefore, dominancebased hierarchies are mainly beneficial for the dominant individuals and their allies. 
104 Therefore, people who do not have a dominant position tend to dislike this type of hierarchy (Brand \& Mesoudi, 2019; Ridgeway \& Diekema, 1989) and, if possible, try to escape the influence of dominant individuals and rebel against them (Boehm et al., 1993; Cheng, 2019; Ridgeway \& Diekema, 1989).

\section{1.- Social Rank Cues}

Because prestige-based and dominance-based hierarchies are associated with beneficial and detrimental fitness consequences respectively in subordinates, it is important for subordinates to identify who is prestigious and who is dominant within a social group. To this end, people use social rank cues or signals that convey information on the level of prestige and dominance of an individual. I classify social rank cues into first-order and second-order cues (Jiménez \& Mesoudi, 2019). Firstorder cues are cues related to the behaviour, appearance, personality, material possessions, etc. of an individual. These can be assessed directly by the observer. Second-order cues are cues related to the behaviour of other people towards an individual and imply, therefore, a more indirect assessment of the individual by the observer.

Prestige cues are cues that people use to infer the competence of an individual within a value domain and the willingness of an individual to provide benefits for the group. Examples of first-order prestige cues are being knowledgeable/skilful (Henrich \& GilWhite, 2001), humble (Cheng, Tracy, \& Henrich, 2010) and showing altruistic behaviour towards the in-group (Halevy, Chou, Cohen, \& Livingston, 2012). Examples of second-order prestige cues are being paid sustained attention with prolonged eye contact, being copied, being popular, and receiving generalised voluntary deference (Henrich \& Gil-White, 2001). 
Dominance cues are cues that people use to infer the fighting skill of an individual and their willingness to use force and intimidation to attain their goals. Examples of firstorder dominant cues are being aggressive/intimidating (Henrich \& Gil-White, 2001), arrogant/narcissist and self-centred (Cheng et al., 2010). Examples of second-order dominance cues are being paid attention but without receiving prolonged eye contact, being obeyed, being disliked/unpopular and receiving generalised coerced deference (Henrich \& Gil-White, 2001).

Medium social rank cues are cues that people use to infer that an individual is not particularly prestigious or dominant but is not at the bottom of either hierarchy. Examples of first-order medium social rank cues are having average knowledge/skill, being modest and regarding highly the contribution of others. Examples of secondorder medium social rank cues are not being paid a lot of attention, not being very influential within a group and receiving deference by only a small group of close friends and relatives.

\section{2.- Hypotheses} Jiménez \& Mesoudi, 2019). However, a key piece in the puzzle of how people learn from prestigious individuals is missing in the literature. That is, there is a lack of studies investigating the cultural transmission of prestige cues themselves. This is an 
important question because it is necessary to identify prestigious individuals to be able to preferentially copy them.

If people select individuals with better-than-average knowledge/skills from whom to learn through the identification of indirect cues that convey high prestige, as Henrich and Gil-White's state, then it is likely than natural selection (or potentially cultural selection, Heyes, 2018) has shaped human cognition to be more attentive to, and process and recall better high prestige social rank cues than social rank cues than descriptions of individuals displaying lower prestige social rank cues.

The identification of individuals who are more likely to impose costs upon others through physical aggression or other types of intimidation might also have been important in human evolution. Natural selection (or cultural selection) might therefore have shaped human cognition to be more attentive, and process and recall better high dominance social rank cues than social rank cues conveying lower dominance. If this is true, then descriptions of individuals displaying high dominance social rank cues would be transmitted with greater fidelity during social interactions than descriptions of individuals displaying lower dominance. Consequently, we predict that both high prestige and high dominance social rank cues are better transmitted than medium social rank cues.

Another important question is whether high dominance cues are better transmitted than high prestige social rank cues, high prestige cues better transmitted than high dominance cues, or both types of high social rank cues are similarly well transmitted. 
choose to transmit more information conveying negative and threat-related information than neutral or positive information (Bebbington et al., 2017; Blaine and Pascal, 2018) and that people are loss aversive, i.e., they prefer not to lose certain amount of money than to gain the same amount of money with the same probability (Kahneman \& Amos, 1979; but see Mukherjee et al., 2017). These pieces of evidence suggest that avoiding costs might have been more important in human evolution than attaining benefits. If so, it is likely that people are more attentive, process, and recall better high dominant social rank cues, which signal the capacity and disposition of an individual to impose costs over others, than high prestige social rank cues, which displays the capacity to provide benefits to others, being injured is likely to have important negative fitness consequences such as impeding physical mobility and difficulties attaining resources. Therefore, high dominance cues, which convey the capacity and willingness to inflict costs upon others should be easily identified and remembered. In contrast, lacking access to the knowledge/skill of a prestigious individual might be less detrimental given the fact that others individuals of the group (e.g., kin) are likely to possess and be willing to share knowledge/skills, which, although less valuable than the knowledge/skills of the prestigious individual, are still likely to be fitness-enhancing. Consequently, we predict that high dominance cues are better transmitted than high prestige cues.

In this experiment, we created fictional descriptions of three footballers playing in three different local football teams, who were described as prestigious, dominant or medium social rank. we use a transmission chain experimental design (Bartlett, 1932; Mesoudi, 2007) in which participants were organised in linear chains of four participants ('cultural generations') in order to test the accumulated effect of memory biases beyond a single individual's recall. Participants in the initial group receive and 
recall the original descriptions, while the remaining participants receive the information recalled by participants in the previous generation of their chain. We preregistered my hypotheses on the Open Science Framework (OSF) website (https://osf.io/68vcs), which were that:

H1: Both high prestige and high dominance cues are more accurately transmitted over experimental cultural generations than medium social rank cues.

H2: High dominance cues are more accurately transmitted over experimental cultural generations than prestige cues.

\section{2.- METHODS}

\section{1.- Ethical Statement}

The study was approved by the Biosciences Ethical Committee at the University of Exeter Cornwall Campus on the 4th November 2019 (Ref eCORN002174 v3.3).

\section{2.- Participants}

Following the procedure stated in the preregistration (https://osf.io/68vcs), we recruited online participants through Prolific (www.prolific.ac) (Peer, Samat, Brandimarte, \& Acquisti, 2016). Using pre-screening filters, we selected participants who were aged 18-60 years, were English native speakers, had British Nationality, had an approval rating on previous Prolific studies of $90 \%$ or above, and had not participated in any of our previous studies through this website.

The data was collected at four different times (one for each "cultural generation"), between the $12^{\text {th }}$ and $19^{\text {th }}$ November 2019. Each time we recruited 30 participants to complete one generation. 30 chains were necessary to provide five replications of 
each of the six counterbalanced versions of the experimental materials (see Section $(£ 1)$.

Following the exclusion criteria stated in the preregistration, we excluded from the dataset the data of two participants who read at least one of the footballers' descriptions at a rate greater than 1080 words/minute. We derived this cut-off point from a previous transmission chain experiment (Jiménez \& Mesoudi, 2019, July 10), in which participants who read the materials faster than this pace were unable to recall anything from the materials. These two excluded participants were replaced by new participants. We also excluded two other participants who occupied the same generation in a chain for which we had already collected a response. As stated in the preregistration, the responses for participants who completed the study first were retained.

Following the procedure stated in the preregistration, we included in the study the data of the participants who first provided a response.

242 Overall, we recruited 124 participants (84 females, 40 males) aged 18-60 (M=37.25, $\mathrm{SD}=10.27$ ), with 120 of these participants (82 females, 38 males) aged 18-60 $(\mathrm{M}=37.44, \mathrm{SD}=10.25)$ included in the analysis.

\subsection{Materials}

Fictional descriptions of three different local footballers (John, Bill and James) who play in different local teams (also fictional) were created as materials to transmit along the chains. These descriptions were created with the intention of describing a prestigious (John), a dominant (Bill) and a medium social rank (James) footballer 
respectively. The materials were based on the dominance-prestige distinction put

251

252

253

254

255

256

257

258

259

260

261

262

263

264

265

266

267

268

269

270

271 forward by Henrich and Gil-White (2001) and Cheng et al. (2010). Following this account, John, our prestigious footballer, was described as a competent footballer who was admired by his teammates. In contrast, Bill, our dominant footballer, was described as a violent footballer who was feared by his teammates. James, our medium social rank footballer, was described as having average football skill without evoking strong emotions. We decided to use medium social rank rather than low social rank because low social ranked individuals were likely to elicit strong emotions of pity and sympathy. Medium social rank is both lacking in such emotions, and in prestige and dominance cues.

The exact descriptions of the three footballers are as follows:

\section{Prestigious footballer (John)}

Everybody in the football team admires John. He is so skilful as a football player that last year he scored the most goals in the local league. Consequently, this year members of the team unanimously voted to make him captain of the team. They also tend to copy whatever he does. At team meetings, the other members always pay careful attention to what he is saying with their eyes fixed on him. Nevertheless, he is modest about his football skills and he always takes other team members' wishes into consideration. Outside of the football team, he is also very popular. People often invite him to parties because they want to spend time with him.

\section{Dominant footballer (Bill)}

Everybody in the football team is afraid of Bill. He is so violent as a football player that last month he injured two teammates during training. This year he self-appointed himself captain without the support of any team member. Other teammates tend to obey him. At team meetings, the other members always pay careful attention to what he is saying, though usually without making eye contact with him. He thinks he is the best player and he never takes other team members' wishes into consideration. 
277 Outside of the football team, people also dislike him, but people invite him to parties because they don't want to make him angry.

Medium social rank footballer (James)

280 People in the football team don't have strong emotions towards James. He is an average football player in the team, scoring only a few goals last year. This year he wanted to be the captain of the team, but he only received two votes. He isn't very influential among his teammates. At team meetings, the other members of the team often don't listen to him very carefully and don't tend to fix their eyes on him for long. He is modest about his football skills, and consequently takes other teammates' perspectives in high regards. Outside the team, he is not particularly popular but he does get invited to parties held by his closest friends because they like to hang out with him.

A within-chain transmission chain design with four generations per chain was used. Participants in the first generation were asked to read and then recall the original descriptions of all three footballers (prestigious, dominant and medium social rank) given above in Section 2.3, although without the labels denoting prestige, dominance or medium social rank. The product of their recall was then transmitted to the next participant in their chain (second generation), who also recalled the material. This procedure was repeated until reaching the fourth generation and across 30 separate, parallel transmission chains to provide independent replications of transmission effects. The order of presentation of the three descriptions was fully counterbalanced, which resulted in six different versions of the experimental materials ( 5 replications for each version). 
303 Participants were first randomly assigned to one of the 30 transmission chains and provided with the following instructions: "In the following you will read three descriptions of three football players who play in different local football teams in the UK. We would like you to read these descriptions very carefully as you will be asked some questions about each of the football players later. It is very important that you read the information at a pace that allows you full comprehension as you will be asked some questions about this information later." Second, they read the three descriptions of the prestigious, dominant and medium social rank footballer. Third, they were asked to provide their basic demographic information (i.e., gender, age, first language, nationality, profession and nearest city from where they live). This served as a distractor task. Fourth, they were asked to recall the descriptions of the three footballers one by one. They were given between a minimum of 1 minute and a maximum of 3 minutes to recall the description of each footballer. Lastly, participants were thanked for their participation and informed about the goal of the experiment and our hypotheses.

319 Participants' recall accuracy was assessed by comparing their correct recall with a preregistered table (https://osf.io/b4nqu/) ${ }^{1}$ containing twelve social rank cues for each description. Examples of prestige cues are to be skilful, admired and copied. Examples of dominance cues are to be violent, feared and obeyed. Examples of medium social rank cues are to have average skill, not to evoke strong emotions and not to be very influential. After data collection, we realised that two cues contained two cues each.

\footnotetext{
${ }^{1}$ The registration form was submitted on $11^{\text {th }}$ November 2019. The data was collected between the $12^{\text {th }}$ and $19^{\text {th }}$ November. During data collection, we realised that the supplementary materials for the preregistration (i.e., experimental materials, table for coding recall and $\mathrm{R}$ script) were not attached to the preregistration form and uploaded again on $15^{\text {th }}$ November 2019.
} 
325 For instance, "being chosen as captain", which in the original table was considered a unique prestige cue could be divided into having the position of captain (being captain) and the process of attainment of the captainship (being chosen/voted). The same was applicable for the equivalent dominance cue ("being self-appointed captain" = being captain + attaining the captainship through self-appointment) and medium social rank cue ("not enough votes for being captain" = not being captain + receiving few votes). The other proposition ("being invited to parties" for both the prestigious and dominant individuals, and "being invited to parties of closest friends" for the medium social rank individual), were considered unfair for the medium social rank individual as it contained more information to remember. Consequently, we changed the recall coding system to accept "being invited to parties" as one cue for the three individuals. An additional proposition specified the people who invited them (i.e., only their friends for the medium social rank individual and the team or people in general for both the prestigious and dominant individual). See Table 1. Both coding systems (the preregistered and the new) yielded qualitatively similar results and, consequently, we only report here the results derived from the new, improved coding system. A second coder, who was blind to the hypothesis, coded $10 \%$ of the chains ( 3 chains) using this improved coding system. A high inter-coding reliability between both coders (Cohen's Kappa $=0.86)$ was found. 


\begin{tabular}{|c|c|c|c|}
\hline PROPOSITIONS & PRESTIGIOUS (John) & DOMINANT (Bill) & MEDIUM SOCIAL RANK (James) \\
\hline $\mathrm{P} 1$ & Is admired & Is feared & Doesn't arouse strong emotions \\
\hline P2 & Skilful & Violent & Average skill \\
\hline P3 & Highest goal score & Injured teammates & Few goals \\
\hline$P 4 A+P 4 B$ & $\begin{array}{l}\text { A: Being captain } \\
\text { B: Being chosen/voted }\end{array}$ & $\begin{array}{l}\text { A: Being captain } \\
\text { B: Being self-appointed }\end{array}$ & $\begin{array}{l}\text { A: Not being captain } \\
\text { B: Not having many votes }\end{array}$ \\
\hline P5 & Is copied & Is obeyed & Isn't very influential \\
\hline P6 & $\begin{array}{l}\text { Is paid attention to / Is } \\
\text { listened to }\end{array}$ & $\begin{array}{l}\text { Is paid attention to / is } \\
\text { listened to }\end{array}$ & $\begin{array}{l}\text { Is not paid (a lot of) attention to / Is not } \\
\text { listened to (very carefully) }\end{array}$ \\
\hline P7 & Received eye contact & People avoid eye contact & Rarely prolonged eye contact \\
\hline P8 & Is modest & $\begin{array}{l}\text { He thinks he is the best } \\
\text { (arrogant/narcissist) }\end{array}$ & Is modest \\
\hline P9 & $\begin{array}{l}\text { Takes others into } \\
\text { consideration }\end{array}$ & $\begin{array}{l}\text { Doesn't take others into } \\
\text { consideration }\end{array}$ & $\begin{array}{l}\text { Take others' perspectives in high } \\
\text { regard (Takes others into } \\
\text { consideration) }\end{array}$ \\
\hline P10 & Is popular / liked & Is unpopular/disliked & isn't particularly popular \\
\hline$P 11 A+P 11 B$ & $\begin{array}{l}\text { A: is invited to parties } \\
\text { B: by the team/people }\end{array}$ & $\begin{array}{l}\text { A: is invited to parties } \\
\text { B: by the team/people }\end{array}$ & $\begin{array}{l}\text { A: is invited to parties } \\
\text { B: by closest friends }\end{array}$ \\
\hline P12 & $\begin{array}{l}\text { People want to spend } \\
\text { more time with him }\end{array}$ & $\begin{array}{l}\text { People don't want to make } \\
\text { him angry }\end{array}$ & $\begin{array}{l}\text { Closest friends like to hang out with } \\
\text { him }\end{array}$ \\
\hline
\end{tabular}

352 All statistical analyses were conducted with Bayesian package brms (Bürkner, 2017) in $\mathrm{R}$ 3.5.3 (R Core Team, 2019). As in our previous transmission chain experiments (Jiménez \& Mesoudi, 2019, Preprint; Jiménez, Stubbersfield, \& Tehrani, 2018), all the regression models were multilevel with intercepts varying by chain. We treated generation as a monotonic variable (Bürkner \& Charpentier, 2018) as recall decreases over generations but the amount of the decrease varies between adjacent generations (Jiménez \& Mesoudi, 2019, July 10). We adopted a model comparison approach, 
model that, in addition to generation, included the type of social rank cue (prestige,

361 dominance, medium social rank) as a predictor. We use leave-one-out cross validation

362 information criterion (LOOIC) (Vehtari, Gabry, \& Gelman, 2019), as a measure of

363 relative model fit. LOOIC can be interpreted similarly to Akaike information criterion

364 (AIC) or Watanabe-Akaike information criterion (WAIC), such that a lower LOOIC 365 indicates better fit to the data.

3.- RESULTS

367 3.1.- Cumulative Recall

368 Figure 1 shows the number of correctly recalled social rank cues across cultural generations for the description of each footballer (prestigious, dominant and medium rank). As in similar transmission chain experiments, the number of correctly recalled

371 social rank cues decreases over cultural generations. As predicted by $\mathrm{H} 1$, the figure

372 clearly shows that the prestige and dominance cues were better transmitted than the medium social rank cues. This effect is present from the first generation, and in each subsequent generation. Contrary to $\mathrm{H} 2$, the figure does not show any clear differences in the transmission of prestige and dominance cues over generations. 


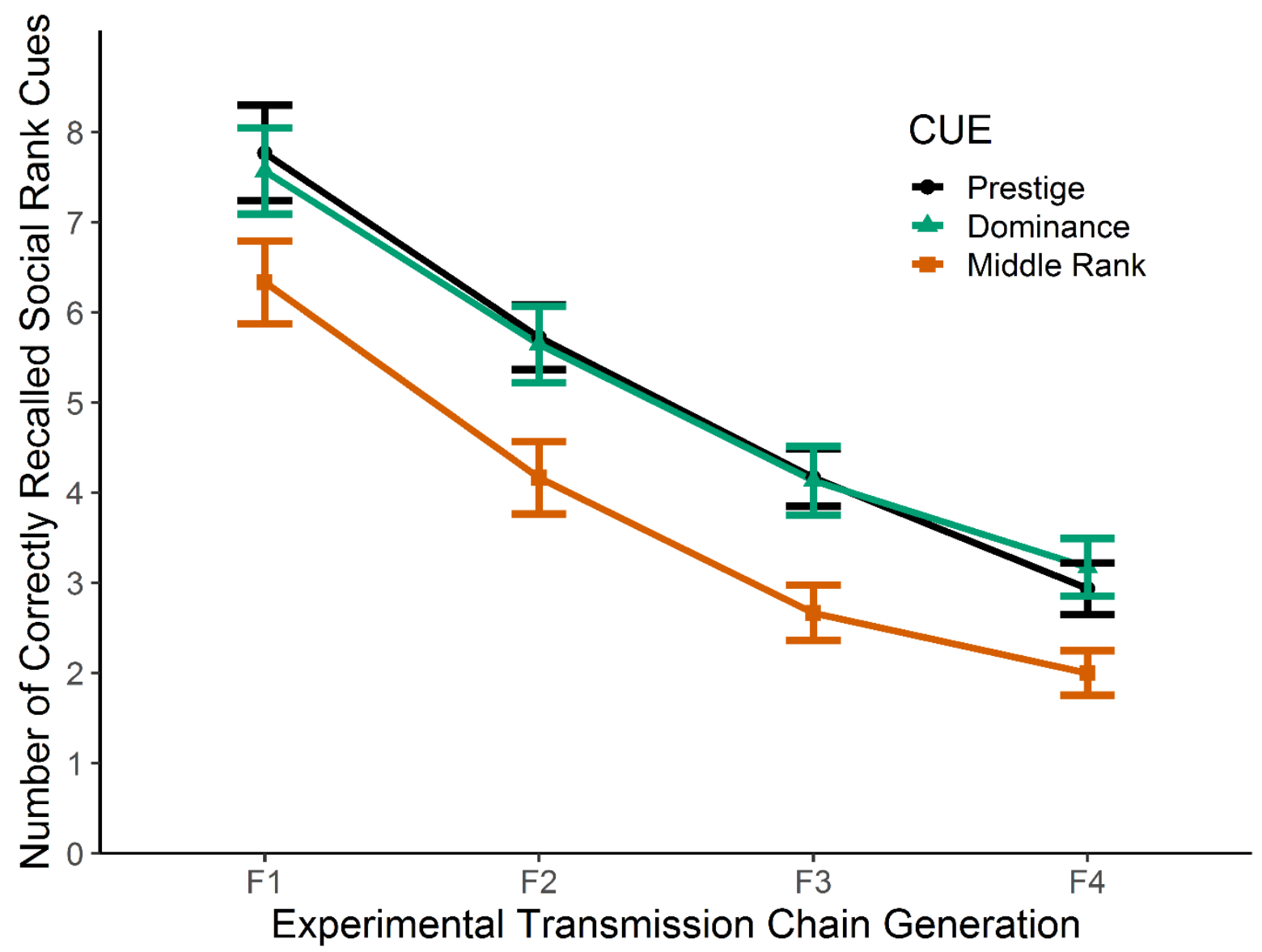

Figure 1. Raw means for the recall of information with 1.60 standard error bars (corresponding to $89 \% \mathrm{Cl}$ as given in the text) plotted against generation. As predicted by H1, both prestige cues and dominance cues were better transmitted than medium social rank cues. Contrary to $\mathrm{H} 2$, there were no clear differences in the transmission of prestige cues and dominance cues over generations.

382 To statistically analyse these trends, we produced several Bayesian multilevel Poisson regression models (See table 1). We first ran a generation-only model that included generation as the sole predictor of recall, which was used as our control model (LOOIC=1431.3, $\mathrm{SE}=17.4)$. We compared this model with our two a priori models. The first was a social rank model, which included the three types of social rank cues (prestige, dominance and medium social rank cues) together with generation as predictors of recall $(\mathrm{LOOIC}=1400.4, \mathrm{SE}=17.5)$. The second was an interaction model, which included the main effects of the social rank cues and generation together with their interaction (LOOIC=1400.9, SE=17.5). Both a priori models had a better fit than the control generation model, with the social rank model being slightly better than the interaction model. As predicted by $\mathrm{H} 1$, both dominance cues $(\mathrm{M}=5.15, \mathrm{SD}=2.75$, 
$394 \mathrm{SE}=0.06,89 \% \mathrm{Cl}[0.21,0.41])$ were better recalled than medium social rank cues the prestige cues $(B=-0.01, S E=0.06,89 \% C I[-0.10,0.09])$.

\begin{tabular}{|c|c|c|c|}
\hline \multirow{3}{*}{$\begin{array}{l}\text { Unstandardized } \\
\text { Coefficients }\end{array}$} & \multicolumn{3}{|c|}{ Main Regression Models } \\
\hline & Generation & Cues & $\begin{array}{c}\text { Cues * } \\
\text { Generation }\end{array}$ \\
\hline & B (SE) & B (SE) & $B(S E)$ \\
\hline Intercept & $1.92(0.08)$ & $\begin{array}{l}1.70 \\
(0.09)\end{array}$ & $1.76(0.10)$ \\
\hline mo(Generation) & $-0.97(0.07)$ & $\begin{array}{l}-0.97 \\
(0.07)\end{array}$ & $-1.12(0.13)$ \\
\hline Cue [Dominance] & & $\begin{array}{c}0.30 \\
(0.06)\end{array}$ & $0.21(0.09)$ \\
\hline Cue [Prestige] & & $\begin{array}{c}0.31 \\
(0.06)\end{array}$ & $0.25(0.08)$ \\
\hline Cue[Dominance]:Generation & & & $0.24(0.17)$ \\
\hline Cue[Prestige]:Generation & & & $0.16(0.17)$ \\
\hline LOOIC & 1431.3 & 1400.4 & 1400.9 \\
\hline model weights & 0.000 & 0.562 & 0.438 \\
\hline
\end{tabular}

Table 2. Unstandardized Coefficients (B) and their standard errors (in brackets) for each of the main regression models. Square brackets indicate the reference categories for the categorical predictors.

Ordinal predictors were modelled as monotonic effects and are labelled mo(variable). More regression models and further details can be found in the Supplementary Materials. LOOIC = leave-one-out cross validation information criterion (lower values indicate better fit to the data; see text for details). Model weights were calculated using pseudo-Bayesian model average weights with Bayesian bootstrap (Vehtari \& Gabry, 2019; Yao, Vehtari, Simpson, \& Gelman, 2018) with the loo package (Vehtari, Gabry, \& Gelman, 2019).

Exploratory analyses were conducted to study the possible effects of gender, age and interest in football on the transmission of social rank cues. The addition of these three variables together or separately to the social rank model did not improve its model fit (see Supplementary Materials). 
To study the narrative evolution, we analysed which social rank cues were well conserved in generation 4 for the description of the prestigious, dominant and medium social rank individuals. We also identified participants' inferences at any generation within the chains and assessed their congruency with the dominance-prestige-medium social rank distinction. These analyses were not included in the preregistration and are exploratory.

For the prestigious individual, the cue that was best conserved in Generation 4 was being popular (67\%), which was followed by being invited to parties (47\%), being the team captain (40\%), being skilful playing football (30\%) and being voted for the captain position (33\%). Participants' inferences mainly referred to his good social skills (e.g., "nice”, "friendly", "kind”, "open”), confidence, competence (e.g., "all round", "golden boy") and the wish of people to be like him. These inferences are all congruent with the notion of a prestigious individual as it was described in the introduction, i.e., as a competent, generous and respected individual.

For the dominant individual, the cue that was best conserved was being feared $(60 \%)$, which was followed by being team captain (43\%), being invited to parties (37\%), being aggressive (33\%), having self-appointed himself the captain position (30\%), being obeyed (30\%) and being unpopular (30\%). Participants' inferences refer to him as an "angry”, "mean”, “nasty”, "unpleasant”, "direct”, "forceful”, "hostile”, "loud” "bully” who "shouts a lot", "is not a good listener" and "people don't' enjoy his company". These inferences are congruent with the notion of a dominant individual as described in the introduction, i.e., an aggressive individual who is feared by people.

For the medium social rank individual, the cue that was best conserved was having average football skill (57\%), followed by being invited to parties (43\%). Participants' 
inferences refer to him as a "good person" (also "nice", "pleasant", who "could be trusted in times of need") with an introverted personality (e.g., "quiet", "shy", "reserved" person who "may lack confidence in larger groups"). These characteristics are compatible with the notion of medium social rank. However, opposite characteristics, i.e., being a bad person with an extroverted personality, would also be compatible with the notion of medium social rank.

\section{4.- DISCUSSION}

In this study, we have tested $(\mathrm{H} 1)$ whether high social rank cues (both prestige and dominance cues) are better transmitted than medium social rank cues and $(\mathrm{H} 2)$ whether dominance cues are better transmitted than prestige cues. To test these two hypotheses, we conducted a transmission chain experiment with four cultural generations. Supporting $\mathrm{H} 1$, we found that both prestige and dominance cues were better transmitted than medium social rank cues. The recall advantage of both high social rank cues was evident in the first generation, which suggests that this effect might be strong enough to be detected in single-generation experiments, although also carried through to all subsequent generations, indicating its long-term stability over repeated transmission episodes. This result is consistent with the evolutionary importance of both prestige-based and dominance-based social hierarchies in human social groups (Henrich \& Gil-White, 2001).

Contrary to $\mathrm{H} 2$, dominance cues were no better transmitted than prestige cues. We predicted a transmission advantage of dominance cues over prestige cues because we assumed that avoiding the potential costs produced by dominant individuals (e.g., physical injuries) were more important than the potential benefits provided by prestigious individuals (e.g., high quality information to socially learn). Our experiment does not test directly this assumption, but the lack of statistical differences between 
the cumulative recall of prestige and dominance cues suggest that identifying both prestigious and dominant individuals might have been equally important in our evolutionary history.

Recall-based transmission chain experiments are generally used to study content transmission biases, i.e., a transmission advantage of information with particular intrinsic characteristics. These studies have identified different content biases such as social (Mesoudi, Whiten, \& Dunbar, 2006; Stubbersfield, Tehrani, \& Flynn, 2014), emotional (Eriksson \& Coultas, 2014; Stubbersfield, Tehrani, \& Flynn, 2017), negative (Bebbington, MacLeod, Ellison, \& Fay, 2017), and stereotypical (Kashima, 2000; Lyons \& Kashima, 2006) content transmission biases. Our results suggest another content transmission bias to add to this list: a social rank content transmission bias. However, the results might also be explained, not by a specific content bias referring to social rank, but by a previously identified content bias: the emotional content bias. Stubbersfield et al. (2017) showed that narratives with high emotional content are better transmitted than narratives with low emotional content. This effect occurred independent of the valence of the emotion (positive as in amusement vs negative as in disgust). It is plausible that our descriptions of both the prestigious individual and the dominant individual have elicited a higher level of emotionality than our description of our medium social rank individual. The description of a prestigious football player might have elicited positive emotions like admiration, while the description of the dominant football player might have elicited negative emotions like fear. This possibility would be congruent with Henrich and Gil-White's dual evolutionary model of social hierarchy, which states that the emotions of admiration and fear elicited in others are the mechanisms by which prestigious and dominant individuals respectively acquired high social rank and influence. As emotions are a proximate mechanism, 
emotional and social rank content biases are not necessarily alternative explanations.

Emotions such as admiration and fear could be the proximate mechanism by which the ultimate-level social rank bias operates. Future studies should use procedures to balance the emotional content across experimental conditions to test whether the elicitation of emotions is the mechanism by which social rank bias operates at a proximate level.

Another possibility is that the experimental materials for both prestige and dominance cues were easier to remember (i.e., less cognitively demanding) than the medium social rank cues due to their higher level of concreteness (Heath \& Heath, 2008). Being at the top of a social hierarchy, being feared or admired, being popular or disliked is more concrete and, consequently, easier to remember, than being in the medium of a social hierarchy, not eliciting strong emotions, or not being particularly popular. Future studies might use a description of an individual at the bottom of both dominance and than the medium social rank description, which might help to alleviate the problem of different levels of emotional content.

In conclusion, we have found evidence to support the higher fidelity transmission of high social rank cues, referring to both prestige and dominance, than medium social rank cues. We found no evidence that supports a transmission advantage of either type of high social rank cue. However, the mechanism that explains these results are not clear. It could be the consequence of a specific content transmission bias referring to high social rank (social rank content transmission bias) or it could be a more general feature of the information such as a higher level of emotional content or concreteness 
of the experimental materials for both the prestigious and dominant individual than for the medium social rank individual. Given that this is the first experiment studying the cultural transmission of social rank cues, we encourage both direct and conceptual

512 replications of this study to further explore the transmission advantage of social rank 513 cues.

515 This research was supported by the Leverhulme Trust (grant RPG-2016-122658 awarded to AM). We thank Rosie Layfield for the second coding of participants' recall, and Rick O'Gorman and Thomas Currie for their comments in earlier versions of this manuscript.

\section{6.- REFERENCES}

520 Anderson, C., Hildreth, J. A. D., \& Howland, L. (2015). Is the Desire for Status a Fundamental Human Motive? A Review of the Empirical Literature. Psychological Bulletin, 141(3), 574-601. doi:10.1037/a0038781

Anderson, C., \& Kilduff, G. J. (2009). Why do dominant personalities attain influence

Anderson, C., \& Willer, R. (2014). Do Status Hierarchies Benefit Groups? A Bounded New York. 
531 Atkisson, C., O'Brien, M. J., \& Mesoudi, A. (2012). Adult learners in a novel environment use prestige-biased social learning. Evolutionary Psychology, 10(3), 519-537.

Bartlett, F. C. (1932). Remembering: a study in experimental and social psychology. Cambridge: Cambridge University Press.

Brand, C. O., Heap, S., Morgan, T. J., \& Mesoudi, A. (2019, December 12). The adaptive use of prestige-biased social learning in an online, knowledge-based task. Preprint at doi.org/10.31234/osf.io/mn9t6 
556 Brand, C. O., \& Mesoudi, A. (2019). Prestige and dominance-based hierarchies exist

557

558

559

560

561

562

563

564

565

566

567

568

569

570

571

572

573

574

575

576

577

578

in naturally occurring human groups, but are unrelated to task-specific knowledge. R Soc Open Sci, 6(5), 181621. doi:10.1098/rsos.181621

Bürkner, P.-C. (2017). brms: An R Package for Bayesian Multilevel Models Using Stan. 2017, 80(1), 28. doi:10.18637/jss.v080.i01

Bürkner, P.-C., \& Charpentier, E. (2018). Monotonic Effects: A Principled Approach for Including Ordinal Predictors in Regression Models. PsyArXiv. doi:10.31234/osf.io/9qkhj

Chagnon, N. (1988). Life Histories, Blood Revenge, and Warfare in a Tribal Population. Science, 239(4843), 985-992. doi:10.1126/science.239.4843.985

Cheng, J. T. (2019). Dominance, Prestige, and the Role of Leveling in Human Social Hierarchy and Equality. Current Opinion in Psychology. doi:10.1016/j.copsyc.2019.10.004

Cheng, J. T., \& Tracy, J. L. (2014). Toward a Unified Science of Hierarchy: Dominance and Prestige are Two Fundamental Pathways to Human Social Rank. 3-27. doi:10.1007/978-1-4939-0867-7_1

Cheng, J. T., Tracy, J. L., Foulsham, T., Kingstone, A., \& Henrich, J. (2013). Two ways to the top: evidence that dominance and prestige are distinct yet viable avenues to social rank and influence. J Pers Soc Psychol, 104(1), 103-125. doi:10.1037/a0030398

Cheng, J. T., Tracy, J. L., \& Henrich, J. (2010). Pride, personality, and the evolutionary foundations of human social status. Evolution and Human Behavior, 31(5), 334347. doi:10.1016/j.evolhumbehav.2010.02.00 
579 Chudek, M., Heller, S., Birch, S., \& Henrich, J. (2012). Prestige-biased cultural

580

581

582

583

584

585

586

587

588

589

590

591

592

593

594

595

596

597

598

599

600

601

602

603 learning: bystander's differential attention to potential models influences children's learning. Evolution and Human Behavior, 33, 46-56

de Waal-Andrews, W., Gregg, A. P., \& Lammers, J. (2015). When status is grabbed and when status is granted: Getting ahead in dominance and prestige hierarchies. British Journal of Social Psychology, 54(3), 445-464. doi:10.1111/bjso.12093

Eriksson, K., \& Coultas, J. C. (2014). Corpses, Maggots, Poodles and Rats: Emotional Selection Operating in Three Phases of Cultural Transmission of Urban Legends. Journal of Cognition and Culture, 14(1-2), 1-26. doi:10.1163/15685373-12342107

Halevy, N., Chou, E. Y., Cohen, T. R., \& Livingston, R. W. (2012). Status Conferral in Intergroup Social Dilemmas: Behavioral Antecedents and Consequences of Prestige and Dominance. Journal of Personality and Social Psychology, 102(2), 351-366. doi:10.1037/a0025515

Heyes, C. (2018). Cognitive Gadgets: The Cultural Evolution of Thinking. Harvard University Press.

Heath, C., \& Heath, D. (2008). Made to stick: why some ideas take hold and others come unstuck. London: London : Arrow Books.

Henrich, J., \& Gil-White, F. J. (2001). The evolution of prestige: freely conferred deference as a mechanism for enhancing the benefits of cultural transmission. Evolution and Human Behavior, 22(3), 165-196. doi:10.1016/S1090$5138(00) 00071-4$

Hill, J. (1984). Prestige and reproductive success in man. Evolution and Human Behavior, 5(2), 77-95. doi:10.1016/0162-3095(84)90011-6 
604 Jiménez, Á. V., \& Mesoudi, A. (2019). Prestige-biased social learning: current

605

606

607

608

609

610

611

612

613

614

615

616

617

618

619

620

621

622

623

624

625

626

627

628

evidence and outstanding questions. Palgrave Communications, 5(1), 20. doi:10.1057/s41599-019-0228-7

Jiménez, Á. V., \& Mesoudi, A. (2019, July 10). Prestige does not affect the cultural transmission of novel controversial arguments in an online transmission chain experiment. Preprint at https://psyarxiv.com/3u9wh

Jiménez, Á. V., \& Mesoudi, A. (2019, December 27). The Integrated Dual Evolutionary Model of Social Hierarchy. Preprint at https://psyarxiv.com/sh7mg/

Jiménez, Á. V., Stubbersfield, J. M., \& Tehrani, J. J. (2018). An experimental investigation into the transmission of antivax attitudes using a fictional health controversy. Social Science \& Medicine, 215, 23-27. doi:10.1016/j.socscimed.2018.08.032

Kahneman, D., \& Tversky, A. (1979). Prospect Theory: An Analysis of Decision under Risk. Econometrica, 47(2), 263-291. doi:10.2307/1914185

Kashima, Y. (2000). Maintaining Cultural Stereotypes in the Serial Reproduction of Narratives. Personality and Social Psychology Bulletin, 26(5), 594-604. doi:10.1177/0146167200267007

Lyons, A., \& Kashima, Y. (2006). Maintaining stereotypes in communication: Investigating memory biases and coherence-seeking in storytelling. Asian Journal of Social Psychology, 9(1), 59-71. doi:10.1111/j.1467839X.2006.00184.x

Maner, J. K., \& Case, C. R. (2016). Chapter Three - Dominance and Prestige: Dual Strategies for Navigating Social Hierarchies. In M. O. James \& P. Z. Mark (Eds.), Advances in Experimental Social Psychology (Vol. Volume 54, pp. 129180): Academic Press. 
Mealey, L. (1985). The relationship between social status and biological success: A case study of the Mormon religious hierarchy. Ethology and Sociobiology, 6(4), 249-257. doi:10.1016/0162-3095(85)90017-2

Mesoudi, A. (2007). Using the methods of experimental social psychology to study cultural evolution. Journal of Social, Evolutionary, and Cultural Psychology, 1(2), 35 .

Mesoudi, A., Whiten, A., \& Dunbar, R. (2006). A bias for social information in human cultural transmission. $\mathrm{Br} \quad J \quad$ Psychol, $\quad 97(\mathrm{Pt} \quad 3), \quad 405-423$. doi:10.1348/000712605×85871

Mukherjee, S., Sahay, A., Pammi, V. S. C., \& Srinivasan, N. (2017). Is loss-aversion magnitude-dependent? Measuring prospective affective judgments regarding gains and losses. Judgment and Decision Making, 12(1), 81-89.

Peer, E., Samat, S., Brandimarte, L., \& Acquisti, A. (2016). Beyond the Turk: an empirical comparison of alternative platforms for crowdsourcing online behavioral research. SSRN. doi:10.2139/ssrn.2594183

Pinker, S. (1998). How the mind works. St Ives: Penguin.

Price, M. E., \& Van Vugt, M. (2014). The evolution of leader-follower reciprocity: the theory of service-for-prestige. Frontiers in Human Neuroscience, 8(363). doi:10.3389/fnhum.2014.00363

R Core Team. (2019). R: A Language and Environment for Statistical Computing. Viena: R Foundation for Statistical Computing. Retrieved from https://www.Rproject.org/

Redhead, D., Cheng, J. T., \& O'Gorman, R. (2018a). Higher Status in Group. In S. T \& W.-S. V (Eds.), Encyclopedia of Evolutionary Psychological Science. New York: Springer. 
654 Redhead, D., Cheng, J. T., \& O'Gorman, R. (2018b). Individuals that Impose Costs.

655

656

657

658

659

660

661

662

663

664

665

666

667

668

669

670

671

672

673

674

675

676
In T. K. Shackelford \& V. A. Weekes-Shackelford (Eds.), Encyclopedia of Evolutionary Psychological Science (pp. 1-6). Cham: Springer International Publishing.

Ridgeway, C. L., \& Diekema, D. (1989). Dominance and collective hierarchy formation in male and female task groups. American Sociological Review, 79-93.

Savin-Williams, R. C. (1979). Dominance Hierarchies in Groups of Early Adolescents. Child Development, 50(4), 923-935. doi:10.2307/1129316

Smith, J. A., \& Foti, R. J. (1998). A pattern approach to the study of leader emergence. The Leadership Quarterly, 9(2), 147-160. doi:10.1016/s1048-9843(98)90002-9

Snyder, J. K., Kirkpatrick, L. A., \& Barrett, H. C. (2008). The dominance dilemma: Do women really prefer dominant mates? Personal Relationships, 15(4), 425-444. doi:10.1111/j.1475-6811.2008.00208.x

Stubbersfield, J. M., Tehrani, J. J., \& Flynn, E. G. (2014). Serial killers, spiders and cybersex: Social and survival information bias in the transmission of urban legends. Br J Psychol, 106(2), 288-307. doi:10.1111/bjop.12073

Stubbersfield, J. M., Tehrani, J. J., \& Flynn, E. G. (2017). Chicken Tumours and a Fishy Revenge: Evidence for Emotional Content Bias in the Cumulative Recall of Urban Legends. Journal of Cognition and Culture, 17(1-2), 12-26. doi:10.1163/15685373-12342189

Vehtari, A., Gabry, J., \& Gelman, A. (2019). loo: Efficient leave-one-out crossvalidation and WAIC for Bayesian models. . Retrieved from https://mcstan.org/loo 
677 Vehtari, A., \& Gabry, J. (2019). Bayesian Stacking and Pseduo-BMA weights using

678

679

680

681

682

683

684

685

686

687

688

689

690

691

692

693

694

695

Retrieved from https://cran.rproject.org/web/packages/loo/vignettes/loo2-weights.html

Von Rueden, C. (2014). The roots and fruits of social status in small-scale human societies. In The psychology of social status (pp. 179-200): Springer.

von Rueden, C., Gurven, M., \& Kaplan, H. (2010). Why do men seek status? Fitness payoffs to dominance and prestige. Proceedings of the Royal Society B: Biological Sciences. doi:10.1098/rspb.2010.2145

von Rueden, C., \& Jaeggi, A. V. (2016). Men's status and reproductive success in 33 nonindustrial societies: Effects of subsistence, marriage system, and reproductive strategy. Proceedings of the National Academy of Sciences of the United States of America, 113(39), 10824-10829. doi:10.1073/pnas. 1606800113

Yao, Y., Vehtari, A., Simpson, D., \& Gelman, A. (2018). Using stacking to average Bayesian predictive distributions (with discussion). Bayesian Analysis, 13(3), 917-1003. 